\title{
Giomer yapıdaki akışkan kompozit ve adeziv sistemin sınıf V kavitelerde mikrosızıntı açısından değerlendirilmesi
}

\author{
Nihan Gönülol, ${ }^{1 *}$ Sezin Özer, ${ }^{2}$ Neslihan Demirel ${ }^{2}$ \\ ${ }^{1}$ Restoratif Diş Tedavisi Anabilim Dalı, ${ }^{2}$ Pedodonti Anabilim \\ Dalı, Ondokuz Mayıs Üniversitesi Diş Hekimliği Fakültesi, \\ Samsun, Türkiye
}

\section{ÖzeT}

AMAÇ: Giomer yapıdaki akışkan kompozit rezin ve adeziv sistemin ve bunların geleneksel yapıdaki akışkan kompozit rezin ve adeziv sistemle kombinasyonunun Sınıf V kavitelerde mikrosızıntı açısından karşılaştırılmasıdır.

GeReç Ve Yöntem: Yirmi adet insan 3. büyük azı dişlerinin bukkal ve lingual yüzeylerine standart Sınıf V kaviteler (mezio-distal genişlik: 4 mm, okluzo-gingival genişlik: $3 \mathrm{~mm}$, derinlik: $2 \mathrm{~mm}$ ) açıldı. Dişler her grupta 10 kavite olacak şekilde rastgele dört gruba ayrıldı. Giomer yapıdaki FL-Bond Il adeziv sistem ve Beautifil Flow Plus akışkan kompozit ile geleneksel yapıdaki Clearfil SE Bond adeziv sistem ve Filtek Ultimate akışkan kompozit kullanılarak dişler şu şekilde restore edildi; Grup FLB: FL-Bond II+Beautifil Flow Plus, Grup FLU: FL-Bond II+Filtek Ultimate, Grup SEB: Clearfil SE Bond+Beautifil Flow Plus, Grup SEU: Clearfil SE Bond+Filtek Ultimate. Dişler, 5-55 ${ }^{\circ} \mathrm{C}$ 'de 1000 kere termal eskitmenin ardından \%0.5 lik bazik fuksin çözeltisi içinde 24 saat süresince bekletildi. Boya sızıntısı stereomikroskop altında değerlendirilerek skorlandı. Verilerin değerlendirilmesinde Kruskal-Wallis Testi ve Mann-Whitney U testleri kullanıldı.

BULGULAR: Gruplar arasında mikrosızıntı değerlerinde hem minede hem de dentinde fark bulundu $(p<0.05)$. Grup FLB, dentinde diğer gruplardan anlamlı bir şekilde daha fazla sızıntı gösterdi $(p<0.05)$. Grup SEU, minede Grup FLB ve Grup SEB'den anlamlı bir şekilde daha fazla sızıntı gösterdi $(p<0.05)$.

Sonuç: Her iki kompozit grubunun, yapısında 10-MDP monomeri içeren geleneksel tipteki adeziv sistemle (Clearfil SE Bond) kombinasyonu dentinde daha az mikrosızıntıya sebep oldu. Minede ise giomer kompozitin uygulandığı gruplarda adezivin tipi fark etmeksizin daha az mikrosızıntı olduğu sonucuna varıldı.

Makale gönderiliş tarihi: 13 Eylül 2013; Yayına kabul tarihi: 29 Kasım 2013 *iletişim: Nihan Gönülol, Restoratif Diş Tedavisi Anabilim Dalı, Ondokuz Mayıs Üniversitesi Diş Hekimliği Fakültesi, Samsun, Türkiye;

e-posta: nihan.gonulol@omu.edu.tr
ANAHTAR Kelimeler: Akışkan hibrid kompozit, florit, giomer, kompozit rezinler, dental sızıntı

Kaynak Göstermek İçin: Gönülol N, Özer S, Demirel N. Giomer yapıdaki akışkan kompozit ve adeziv sistemin Sınıf V kavitelerde mikrosızıntı açısından değerlendirilmesi. Acta Odontol Turc 2014;31(1):18-22

[Abstract in English is at the end of the manuscript]

\section{Giriş}

Günümüzde sekonder çürükler restoratif diş hekimliğinde çok sık karşılaşılan ve halen çözülememiş bir sorundur. Bu sorunu çözmek amacıyla kompozit rezinlerin rezin matriks ve doldurucu içeriklerinde çeşitli modifikasyonlar yapılarak antibakteriyel aktivitenin sağlanmaSı ve sekonder çürüklerin önlenmesi amaçlanmıştır. ${ }^{1}$ Geleneksel cam ionomer simanlar, florid salınım özelliklerinden dolayı remineralizasyon oluşumunu destekleyip karyojenik bakterilerin metabolizmasını ve büyümesini inhibe ederek çürük oluşumunu engellemektedirler. ${ }^{2,3}$ Bununla birlikte, mekanik özelliklerinin kompozit rezinlere oranla oldukça zayıf olması, okluzal kuvvetlerin yoğun olduğu bölgelerde kullanımlarını kısıtlamaktadır. ${ }^{4}$ Cam iyonomer simanların florid salınım özellikleri ile kompozit rezinlerin estetik ve mekanik özelliklerinin kombine edilmesi amacıyla rezin modifiye cam ionomer simanlar ve poliasit modifiye kompozit rezin gibi hibrit restoratif materyaller geliştirilmiştir. ${ }^{5}$ Son yıllarda ise yeni grup hibrit kompozit materyaller olan giomerler piyasaya sürülmüştür. Bu materyallerde restoratif materyal içerisinde stabil halde cam ionomer üretmek için önceden tepkimeye girmiş cam ionomer teknolojisi (S-PRG) kullanılmaktadır. Flor-alumina silikat cam, polialkenoik asit ile suda reaksiyona girdikten sonra silika dolduruculu üretan rezin içerisine katılırlar. Bu materyallerin florid salma ve depolama özellikleri vardır. Ayrıca üstün estetik özellikleri, kolay cilalanabilmeleri ve kompozit rezinler kadar dirençli olmaları gibi avantajları mevcuttur. ${ }^{6}$ Bu sistemlerin aynı zamanda dentin adeziv sistemleri de üretilmiştir ve benzer şekilde florid salabilme ve depolama özelliklerine sahiptirler. 
Akışkan kompozitler ilk olarak 1990 'lı yılların ortalarında piyasaya sürülmüştür ve restoratif uygulamalarda geniş bir kullanım alanına sahiptir. ${ }^{7}$ Doldurucu boyutları hibrit kompozitlere benzemekle birlikte oranları daha düşük olduğundan viskoziteleri ve elastisite modülleri de daha düşüktür. ${ }^{8}$ Akışkan kompozitler, Sınıf I ve Sınıf II kavitelerde astar materyali olarak kullanılırken Sınıf V kavitelerde daimi restorasyon materyali olarak kullanımları önerilmektedir. ${ }^{9}$

Bu çalışmanın amacı S-PRG teknoloji ile üretilen giomer yapıdaki akışkan bir kompozit rezin ve adeziv sistemin ve bunların geleneksel yapıdaki akışkan kompozit ve adeziv sistemle kombinasyonunun Sınıf $V$ kavitelerde mikrosızıntı açısından karşılaştırılmasıdır.

Çalışmamızın sıfır hipotezi; karşılaştııılan akışkan kompozit rezinler ve adeziv sistemler arasında mine ve dentinde mikrosızıntı değerleri açısından fark olmayacağıdır.

\section{Gereç Ve Yöntem}

Bu çalışmada 20 adet yeni çekilmiş sağlam insan 3 . büyük azı dişi kullanıldı. Çekim sonrasında kök yüzeylerindeki artık dokular kretuar yardımıyla uzaklaştııııp, dişler pomza ve politür fırçasıyla temizlendi. Dişler distile suda oda sıcaklığında muhafaza edildi. Dişlerin bukkal ve lingual yüzeylerine standart Sınıf $V$ kaviteler (mesio-distal genişlik: $4 \mathrm{~mm}$, okluzo-gingival genişlik: 3 $\mathrm{mm}$ ve derinlik: $2 \mathrm{~mm}$ ) su soğutması altında silindirik elmas frezlerle hazırlandı. Gingival kenarlar mine-sement sınırının $1 \mathrm{~mm}$ altında, okluzal kenarlar ise bu sınırın 2 mm üzerinde hazırlandı. Kavite kenarlarına bizotaj yapılmadı. Her beş kavite preparasyonundan sonra yeni frez kullanıldı. Daha sonra dişler her grupta 10 kavite olacak şekilde rastgele 4 gruba ayrıldı. Çalışmada kullanılan materyaller ve özellikleri Tablo 1'de belirtilmiştir.

Grup FLB: Hazırlanan kavite yüzeylerine giomer yapıdaki iki aşamalı kendinden pürüzlendirmeli adeziv sistem olan FL-Bond II üretici önerileri doğrultusunda uygulandı. Önce primer uygulanıp $10 \mathrm{sn}$ bekletildi, havayla kurutuldu. Ardından bonding ajan uygulanıp $10 \mathrm{sn}$ LED ışık cihazı (Elipar FreeLight 2, 3M ESPE, St. Paul, MN, ABD) ile polimerize edildi. Giomer yapıdaki Beautifil Flow Plus akışkan kompoziti tek tabaka halinde kaviteye uygulanarak $10 \mathrm{sn}$ polimerize edildi.

Grup FLU: Hazırlanan kavite yüzeylerine FL-Bond II üretici önerileri doğrultusunda uygulanıp polimerize edildikten sonra geleneksel yapıdaki Filtek Ultimate akışkan kompozit tek tabaka halinde kaviteye uygulanarak $10 \mathrm{sn}$ polimerize edildi.

Grup SEB: Hazırlanan kavite yüzeylerine geleneksel yapıdaki iki aşamalı kendinden pürüzlendirmeli adeziv sistem olan Clearfil SE Bond üretici önerileri doğrultusunda uygulandı. Önce primer uygulanıp 20 sn bekletildi, havayla kurutuldu. Ardından bonding ajan uygulanıp havayla hafifçe kurutularak 10 sn polimerize edildi. Daha sonra Beautifil Flow Plus tek tabaka halinde kaviteye uygulanarak 10 sn polimerize edildi.

Grup SEU: Hazırlanan kavite yüzeylerine Clearfil SE Bond üretici önerileri doğrultusunda uygulandı. Filtek UItimate akışkan kompoziti tek tabaka halinde kaviteye uygulanarak 10 sn polimerize edildi.

Tablo 1. Çalışmada kullanılan materyaller ve özellikleri

\begin{tabular}{|c|c|c|c|}
\hline Materyaller & Bileşimi & Lot no & Üretici firma \\
\hline $\begin{array}{l}\text { FL-Bond II } \\
\text { (2 aşamalı kendinden pürüzlendirmeli } \\
\text { adeziv sistem; giomer yapı) }\end{array}$ & $\begin{array}{l}\text { Primer; su, etanol, karboksilik asit monomer, } \\
\text { fosforik asit monomer, başlatıcı } \\
\text { Bonding ajan; S-PRG dolduruculu } \\
\text { fluoroboroaluminasilikat cam, UDMA, TEGDMA, } \\
\text { 2-HEMA, başlatıcı }\end{array}$ & 071201 & Shofu Inc. Kyoto, Japonya \\
\hline $\begin{array}{l}\text { Beautifil Flow Plus } \\
\text { (A2; akışkan kompozit rezin; } \\
\text { giomer yapı) }\end{array}$ & $\begin{array}{l}\text { Bis-GMA/TEGDMA rezin, S-PRG dolduruculu } \\
\text { fluoroboroaluminasilikat Doldurucu oranı; } \\
\% 66.8 \text { (ağırlık), \%46.3 (hacim) }\end{array}$ & 081213 & Shofu Inc. Kyoto, Japonya \\
\hline $\begin{array}{l}\text { Clearfil SE Bond } \\
\text { ( } 2 \text { aşamalı kendinden pürüzlendirmeli } \\
\text { adeziv sistem) }\end{array}$ & $\begin{array}{l}\text { Primer; MDP, HEMA, hidrofilik alifatik dimetakrilat, } \\
\text { di-kamforokinon, N,N-dietanol p-toluidin, su } \\
\text { Bond; MDP, Bis-GMA, HEMA, } \\
\text { hidrofobik alifatik dimetakrilat, di- kamforokinon, } \\
\text { N-dietanol p-toluidin, kolloidal silika }\end{array}$ & 041819 & $\begin{array}{l}\text { Kuraray Medical Inc, Okayama, } \\
\text { Japonya }\end{array}$ \\
\hline $\begin{array}{l}\text { Filtek Ultimate } \\
\text { (A2; akışkan kompozit rezin) }\end{array}$ & $\begin{array}{l}\text { Bis-GMA, TEGDMA, Prokrilat rezin, } \\
\text { nanoboyutta silika, zirkonia öbekler ve } \\
\text { ytterbium trifluorid. Doldurucu oranı; } \\
\% 65 \text { (ağırlık), \%46 (hacim) }\end{array}$ & N474058 & 3M ESPE, St.Paul, MN, ABD \\
\hline
\end{tabular}


Bütün işlemler aynı araştırmacı tarafından yapıldı. Restorasyonlar tamamlandıktan sonra alüminyum oksit kaplı diskler ile (Sof-Lex, 3M ESPE) bitirme ve polisaj işlemleri tamamlandı. Daha sonra dişler 5-55 ${ }^{\circ} \mathrm{C}$ 'lerde 30 sn bekleme süresiyle 1000 kere termal eskitmeye tabii tutuldu (DTS B1, Dentester, Salubris Technica, İstanbul, Türkiye). Termal eskitme sonrasında restorasyonların $1 \mathrm{~mm}$ yakınlarına kadar olan yerler açıkta kalacak şekilde, dişlerin tüm yüzeylerine 2 kat tırnak cilası uygulandı ve kök uçları mumla kapatıldı. Dişler \% 0.5 bazik fuksin çözeltisi içinde 24 sa süreyle oda sıcaklığında bekletildi. Akar suda yıkanan dişler bukko-lingual doğrultuda elmas separe ile (Isomet, Buehler Ltd, Lake Bluff, IL, ABD) dikey yönde ikiye ayrıldı. Boya sızıntısı $\times 40$ büyütmede stereomikroskop altında değerlendirilerek (Nikon SMZ 1500, Tokyo, Japonya) ve iki farklı araştırmacı tarafından konsensus oluşturularak aşağıdaki şekilde skorlandı;

$0=$ Boya sızıntısı yok .

1= Boya sızıntısı servikal/okluzal kavite duvarının yarısına kadar uzanıyor.

$2=$ Boya sızıntısı servikal/okluzal kavite duvarının yarısından ileriye uzanıyor.

$3=$ Aksiyal duvar boyunca boya sızıntısı var.

Verilerin istatistiksel değerlendirilmesinde KruskalWallis ve Mann-Whitney U testleri kullanılmıştır.

\section{BULGULAR}

Mine ve dentin için mikrosızıntı skorlarının dağııımı Tablo 2'de gösterilmiştir. Kruskal-Wallis testi sonuçlarına göre test edilen gruplar arasında mikrosızıntı değerleri açısından hem minede $(p=0.042)$ hem de dentinde fark tespit edilmiştir ( $p=0.000$; Tablo 3 ). Grup FLB diğer gruplardan istatistiksel olarak anlamlı bir şekilde dentinde daha fazla sızıntı göstermiştir $(p<0.05)$. Minede ise Grup SEU, Grup SEB ve Grup FLB'den istatistiksel olarak anlamlı bir şekilde daha fazla sızıntı göstermiştir $(p<0.05)$.

Grup FLB'de mine ve dentinde mikrosızıntı değerleri açısından fark vardır ve dentinde daha yüksektir $(p=0.004)$. FLU ve SEB gruplarında mine ve dentinde

Tablo 2. Mine ve dentin kenarları için mikrosızıntı skorlarının dağıımı $(n=10)$

\begin{tabular}{lllllllll}
\hline & \multicolumn{4}{c}{ Mine } & \multicolumn{5}{c}{ Dentin } \\
& $\mathbf{0}$ & $\mathbf{1}$ & $\mathbf{2}$ & $\mathbf{3}$ & $\mathbf{0}$ & $\mathbf{1}$ & $\mathbf{2}$ & $\mathbf{3}$ \\
\hline Grup FLB & 6 & 4 & 0 & 0 & 0 & 1 & 1 & 8 \\
Grup FLU & 3 & 7 & 0 & 0 & 4 & 4 & 1 & 1 \\
Grup SEB & 6 & 4 & 0 & 0 & 8 & 1 & 0 & 1 \\
Grup SEU & 1 & 8 & 1 & 0 & 6 & 4 & 0 & 0 \\
\hline
\end{tabular}

Tüm hakları sakııdır @ 2014 Gazi Üniversitesi
Tablo 3. Kruskal-Wallis test sonuçlarına göre deney gruplarının karşılaştırılması

\begin{tabular}{lccccc}
\hline Mine & $\mathbf{n}$ & mean rank & medyan & ki-kare & p değeri \\
\hline Grup FLB & 10 & 16.30 & 0 & 8.193 & 0.042 \\
Grup FLU & 10 & 22.15 & 1 & & \\
Grup SEB & 10 & 16.30 & 0 & & \\
Grup SEU & 10 & 27.25 & 1 & & \\
Dentin & $\mathbf{n}$ & mean rank & medyan & ki-kare & p değeri \\
Grup FLB & 10 & 33.70 & 3 & 21.087 & 0.000 \\
Grup FLU & 10 & 19.70 & 1 & & \\
Grup SEB & 10 & 13.50 & 0 & & \\
Grup SEU & 10 & 15.10 & 0 & & \\
\hline
\end{tabular}

mikrosızıntı değerleri açısından fark yoktur. Grup SEU'da ise mine ve dentinde mikrosızıntı değerleri açısından fark vardır ve minede daha yüksektir $(\mathrm{p}=0.014)$.

\section{TARTIŞMA}

Çalışmamız sonucunda, karşılaştırılan akışkan kompozit rezinler ve adeziv sistemler arasında mine ve dentinde mikrosızıntı değerleri açısından fark olmayacağı yönündeki sıfır hipotezimiz reddedilmiştir.

Kompozit rezin restorasyonların klinik başarısında diş/restorasyon ara yüzündeki mikrosızıntının minimalize edilmesi oldukça önemlidir. Restorasyonların bu yöndeki başarısızlıkları kenar renklenmelerine, pulpal reaksiyonlara, post-operatif hassasiyete ve sekonder çürüklere neden olmaktadır. ${ }^{10}$

Mikrosızıntı testi, klinisyenler ve araştırmacılar tarafından restoratif materyallerin performansının ölçülmesinde sıklıkla kullanılan bir yöntemdir. Bu amaçla en sık kullanılan yöntem, diş ile restorasyon arasındaki boya sızıntısının incelenmesidir. Bu yöntemin hızı, basit ve ekonomik olması gibi avantajlarından dolayı çalışmamızda bu yöntem kullanılmıştır. ${ }^{11,12}$

Akışkan kompozitler üretildikleri günden günümüze kadar astar maddesi, kaide maddesi, build-up materyali, daimi restorasyon materyali veya fissür örtücü gibi geniş kullanım alanları bulmuşlardır. ${ }^{7}$ Bazı çalışmalarda astar/kaide maddesi olarak kullanıldıklarında akışkan kompozitlerin, bonding ajanlarını restoratif materyalin polimerizasyon stresinden koruduğu belirtilirken, ${ }^{13,14}$ bazılarında ise astar maddesi olarak kullanımlarının marjinal mikrosızıntının azalmasına belirgin bir katkısının olmadığı bildirilmiştir. ${ }^{15,16} \mathrm{Bu}$ çalışmada akışkan kompozitler daimi restorasyon materyali olarak Sınıf $\mathrm{V}$ kavitelere tek kütle halinde yerleştirilmiştir.

Giomerler, cam ionomer-kompozit kombinasyonu ile oluşan hibrit materyallerin en güncel olanıdır. Giomerler diğer kompozit rezinler gibi diş dokusuna bağlanmak 
için bonding ajan gerektirirler, ışıkla aktive olurlar, kullanımları kolaydır ve cam ionomerler gibi flor salabilirler. ${ }^{17}$ Yapılan çalışmalarda giomerlerin yüksek florid salabilme ve depolama özelliklerinden dolayı sekonder çürükleri ve demineralizasyonu \%14-35 oranında azalttığı bildirilmiştir. ${ }^{18,19}$ Bu kompozit restoratif materyallerle ilgili yapılan çalışmalar incelendiğinde diş dokusuna bağlantısı için üretici önerileri doğrultusunda yine florid depolayabilen adeziv sistemlerle birlikte kullanıldığı tespit edilmiştir. ${ }^{20-22} \mathrm{Bu}$ çalışmada ise florid salan ve depolayabilen bu materyallerin geleneksel tipteki kompozit rezin ve adeziv sistemle kombinasyonunun mikrosızıntı açısından karşılaştırmalı olarak incelenmesi amaçlanmiştır.

Son yıllarda kendinden pürüzlendirmeli adeziv sistemlerin kullanımı oldukça yaygınlaşmıştır. Asit ve primerin bir sistem içinde birlikte kullanımı hem uygulama süresini kısaltır hem de teknik hassasiyeti azaltır. Bunun yanında kendinden pürüzlendirme sistemlerin mineye bağlantısı ile ilgili çelişkiler devam etmektedir. ${ }^{10} \mathrm{Bu}$ çalışmada da tüm gruplarda restoratif materyalin dişe bağlantısı için kendinden pürüzlendirmeli adezivler kullanılmış ve Grup FLB dışındaki tüm gruplarda minedeki mikrosızıntı değerleri dentine göre daha yüksek tespit edilmiştir.

Çalışmamızda FL Bond II adeziv sistemin Beautifil Flow Plus ile birlikte kullanıldığı grupta dentinde diğer gruplara göre daha yüksek mikrosızıntı değerleri elde edilmiştir. Çalışmamızda kullanılan Clearfil SE Bond'un pH değeri 2'ye yakın iken FL Bond II adeziv sistemin pH değeri 2.4'tür ve her iki adeziv de orta asiditede kendinden pürüzlendirme adezivlerdir. Orta asiditedeki kendinden pürüzlendirmeli adezivlerin total-etch sistemlere göre daha ince hibrid tabakası oluşturduğu bildirilmiştir. Daha az dentin demineralizasyonu oluştuğundan, smear tabakası dentinal tübül girişlerini tıkar, rezin kısmi olarak infiltre olur ve daha az rezin çubukları meydana gelir. Ancak Clearfil SE Bond içerisindeki 10-metakriloiloksidesil dihidrojen fosfat (10-MDP) fonksiyonel monomerinin iki hidroksil grubu vardır ve kalsiyuma bağlanır. 10-MDP fonksiyonel monomeri, diğer monomerlerin demineralize dentine penetrasyonu ve polimerizasyonu kolaylaştırarak kısmen daha kalın bir hibrit tabaka oluşmasını sağlar. ${ }^{23}$ Yoshida ve ark. ${ }^{23}$ çalışmalarında Clearfil SE Bond'un kimyasal yapısının farklılığından dolayı diğer kendinden pürüzlendirmeli adezivlere oranla daha az boya penetrasyonuna neden olduğunu bildirmişlerdir. Bizim çalışmamızda da benzer olarak dentinde Grup SEB ve Grup SEU'da Grup FLB'den daha az mikrosızıntı tespit edilmiştir.

Grup FLU ile Grup SEB ve SEU grupları arasında da fark bulunamamıştır. Bunun nedeni Filtek Ultimate'in monomer yapısına bağlı olduğunu düşünmekteyiz. Fil- tek Ultimate akışkan kompozitin yapısında yeni geliştirilen prokrilat monomeri bulunmaktadır. TEGDMA monomeri düşük viskoziteli olup daha fazla polimerizasyon büzülmesine neden olmaktadır. Polimerizasyon büzülmesini azaltmak amacıyla prokrilat monomeri geliştirilmiştir ve bu monomer yüksek molekül ağırlığının yanında düşük viskoziteye sahiptir. Dolayısıyla bileşik içerisinde TEGDMA oranın azalması bu kompozitlerin polimerizasyon büzülmesini azaltmaktadır. ${ }^{24}$ Dolayısıyla çalışmamızda da Filtek Ultimate akışkan kompozitin FL Bond II ile birlikte kullanıldığı grupta Beautifil Flow Plus grubuna göre daha az mikrosızıntı göstermesi bu nedene bağlı olabilir.

\section{SonUÇ}

Hem giomer yapıdaki hem de geleneksel yapıdaki akışkan kompozitlerin, yapısında 10-MDP monomeri içeren geleneksel tipteki adeziv sistemle (Clearfil SE Bond) kombinasyonunun dentinde daha az mikrosızıntıya sebep olduğu tespit edilmiştir. Minede ise giomer kompozitin uygulandığı gruplarda adezivin tipi fark etmeksizin daha az mikrosızıntı olduğu sonucuna varılmıştır.

Çıkar çatışması: Yazarlar bu çalışmayla ilgili herhangi bir çıkar çatışmalarının bulunmadığını bildirmişlerdir.

\section{KAYNAKLAR}

1. Saku S, Kotake H, Scougall-Vilchis RJ, Ohashi S, Hotta M, Horiuchi $\mathrm{S}$, et al. Antibacterial activity of composite resin with glass-ionomer filler particles. Dent Mater J 2010;29:193-8.

2. Seppä L, Torppa-Saarinen E, Luoma H. Effect of different glass ionomers on the acid production and electrolyte metabolism of Streptococcus mutans Ingbritt. Caries Res 1992;26:434-8.

3. Wiegand A, Buchalla W, Attin T. Review on fluoride-releasing restorative materials--fluoride release and uptake characteristics, antibacterial activity and influence on caries formation. Dent Mater 2007;23:343-62.

4. Papacchini F, Goracci C, Sadek FT, Monticelli F, Garcia-Godoy F, Ferrari M. Microtensile bond strength to ground enamel by glass-ionomers, resin-modified glass-ionomers, and resin composites used as pit and fissure sealants. J Dent 2005;33:459-67.

5. Tian F, Yap AU, Wang X, Gao X. Effect of staining solutions on color of pre-reacted glass-ionomer containing composites. Dent Mater J 2012;31:384-8.

6. Kimyai S, Savadi-Oskoee S, Ajami AA, Sadr A, Asdagh S. Effect of three prophylaxis methods on surface roughness of giomer. Med Oral Patol Oral Cir Bucal 2011;16:e110-4.

7. Bonilla ED, Stevenson RG, Caputo AA, White SN. Microleakage resistance of minimally invasive Class I flowable composite restorations. Oper Dent 2012;37:290-8.

8. Awliya WY, El-Sahn AM. Leakage pathway of Class $V$ cavities restored with different flowable resin composite restorations. Oper Dent 2008;33:31-6.

9. Kugel G, Perry R. Direct composite resins: an update. Compend Contin Educ Dent 2002;23:593-8. 
10. Siso HS, Kustarci A, Göktolga EG. Microleakage in resin composite restorations after antimicrobial pre-treatments: effect of KTP laser, chlorhexidine gluconate and Clearfil Protect Bond. Oper Dent 2009;34:321-7.

11. Nilgun Ozturk A, Usumez A, Ozturk B, Usumez S. Influence of different light sources on microleakage of class $\mathrm{V}$ composite resin restorations. J Oral Rehabil 2004;31:500-4.

12. Arisu HD, Uçtasli MB, Eligüzeloglu $E$, Ozcan $S$, Omürlü $H$. The effect of occlusal loading on the microleakage of Class $\mathrm{V}$ restorations. Oper Dent 2008;33:135-41.

13. Kemp-Scholte CM, Davidson CL. Marginal sealing of curing contraction gaps in Class $\mathrm{V}$ composite resin restorations. J Dent Res 1988;67:841-5

14. Kemp-Scholte CM, Davidson CL. Complete marginal seal of Class $\mathrm{V}$ resin composite restorations effected by increased flexibility. J Dent Res 1990;69:1240-3.

15. Jain $P$, Belcher M. Microleakage of Class II resin-based composite restorations with flowable composite in the proximal box. Am J Dent 2000;13:235-8

16. Chuang SF, Liu JK, Chao CC, Liao FP, Chen YH. Effects of flowable composite lining and operator experience on microleakage and internal voids in Class II composite restorations. J Prosthet Dent 2001;85:17783

17. Kooi TJ, Tan QZ, Yap AU, Guo W, Tay KJ, Soh MS. Effects of foodsimulating liquids on surface properties of giomer restoratives. Oper Dent 2012;37:665-71.

18. Naoum S, Ellakwa A, Martin F, Swain M. Fluoride release, recharge and mechanical property stability of various fluoride-containing resin composites. Oper Dent 2011;36:422-32.

19. Dionysopoulos P, Kotsanos N, Koliniotou-Koubia E, Tolidis K. Inhibition of demineralization in vitro around fluoride releasing materials. $J$ Oral Rehabil 2003;30:1216-22.

20. Sabatini C. Effect of phosphoric acid etching on the shear bond strength of two self-etch adhesives. J Appl Oral Sci 2013;21:56-62.

21. Naoum S, O'Regan J, Ellakwa A, Benkhart R, Swain M, Martin E. The effect of repeated fluoride recharge and storage media on bond durability of fluoride rechargeable Giomer bonding agent. Aust Dent $J$ 2012;57:178-83.

22. Gordan VV, Mondragon E, Watson RE, Garvan C, Mjör IA. A clinical evaluation of a self-etching primer and a giomer restorative material: results at eight years. J Am Dent Assoc 2007;138:621-7.

23. Yoshida Y, Nagakane K, Fukuda R, Nakayama Y, Okazaki M, Shintani $\mathrm{H}$, et al. Comparative study on adhesive performance of functional monomers. J Dent Res 2004;83:454-8.

24. Filtek Ultimate Flowable Restorative technical product profile (3M ESPE); p.6-7.

\section{Microleakage evaluation of giomer flowable composites and adhesive systems in Class V cavities}

\section{Abstract}

OBJeCtive: To evaluate the microleakage of a giomer-based flowable composite resin and an adhesive system, and their combination with a conventional flowable composite resin and an adhesive system in Class $\mathrm{V}$ cavities.

Materials AND Method: Standardized Class V cavities (mesial-distal width: $4 \mathrm{~mm}$, occlusal-gingival width: $3 \mathrm{~mm}$, depth: $2 \mathrm{~mm}$ ) were prepared on the buccal and lingual sides of 20 human third molars. The teeth were randomly divided into 4 groups containing 10 cavities each. Giomer adhesive system FL-Bond II and flowable composite Beautifil Flow Plus and conventional adhesive system Clearfil SE Bond and flowable composite Filtek Ultimate were used as follows; Group FLB: FL-Bond II+Beautifil Flow Plus, Group FLU: FL-Bond II+Filtek Ultimate, Group SEB: Clearfil SE Bond+Beautifil Flow Plus, Group SEU: Clearfil SE Bond+Filtek Ultimate. The teeth were subjected to 1000 thermal cycles between $5-55{ }^{\circ} \mathrm{C}$ and then immersed in $0.5 \%$ basic fuchsin solution for $\mathbf{2 4} \mathrm{h}$. The dye penetration was examined under a stereomisroscope and scored. The results were statistically analyzed by using Kruskal-Wallis and Mann-Whitney U tests.

RESULTS: Significant differences were detected both in enamel and in dentin $(p<0.05)$. In dentin, Group FLB exhibited significantly higher microleakage scores than the other groups $(p<0.05)$. In enamel, Group SEU exhibited significantly higher microleakage scores than Group FLB and Group SEB ( $p<0.05)$.

CONCLUSION: In dentin, combination of both type of flowable composites with the 10-MDP-containing conventional adhesive system (Clearfil SE Bond) caused less microleakage. In enamel, regardless of the type of the adhesive system groups restored with the giomer composite exhibited less microleakage.

KEYWORDS: Composite resins; dental leakage; flowable hybrid composite; fluorid, giomer 